\title{
Reduction and Renormalization
}

\author{
Robert W. Batterman \\ University of Western Ontario
}

July 28, 2006 


\section{Introduction}

In this paper I want to consider the so-called reduction of thermodynamics to statistical mechanics from both historical and relatively contemporary points of view. As is well known, most philosophers not working in the foundations of statistical physics still take this reduction to be a paradigm instance of that type of intertheoretic relation. However, numerous careful investigations by many philosophers of physics and physicists with philosophical tendencies show this view is by and large mistaken. It is almost surely the case that thermodynamics does not reduce to statistical mechanics according to the received view of the nature of reduction in the philosophical literature. What is interesting is that, while not framing the issue in these terms, J. Willard Gibbs can also be seen as being somewhat sceptical about the possibility of a philosophical reduction of thermodynamics to statistical mechanics. Gibbs' scepticism is, of course well-known. Nevertheless, I think his remarks bear further consideration given certain advances in understanding the foundations of statistical physics.

I will first briefly run over some philosophical ground, outlining the received approach to theory reduction as well as what I take to be a more promising conception of reduction that parallels, to some extent the way physicists typically speak of theory reduction. Following this I will discuss Gibbs' famous caution in connecting thermodynamical concepts with those from statistical mechanics. This is presented in chapter XIV, "Discussion of Thermodynamic Analogies," of his book Elementary Principles in Statistical Mechanics. We will see that there are several reasons Gibbs held back from identifying thermodynamic quantities such as temperature and entropy with specific statistical mechanical quantities. I will then present a sketch of a program for reduction that involves deep connections between results in probability theory on limit theorems and the so-called real space renormalization techniques that play such an essential role in understanding the universality of critical phenomena. The framework provides a way of unifying two fundamental problems - the so-called equivalence of ensembles and the existence of critical phases. 


\section{Philosophical Reduction}

Most contemporary views about reduction owe much to the seminal work of Ernest Nagel. In The Structure of Science Nagel asserts that "[r]eduction ... is the explanation of a theory or a set of experimental laws established in one area of inquiry, by a theory usually though not invariably formulated for some other domain." [10, p. 338] Standard views about explanation hold that to explain some phenomenon requires the derivation of a statement characterizing that phenomenon from laws of some true theory. Thus, reduction, very roughly, involves the derivation of the laws of the "reduced" theory from the laws of the "reducing" theory. In the case of thermodynamics we have a reduction to statistical mechanics because the laws of thermodynamics are (supposedly) derivable from those of statistical mechanics.

Of course, Nagel himself realized that to actually carry out such derivations requires nontrivial work. Here is one major problem: In most cases the laws of the reduced theory (thermodynamics) contain terms that do not appear in the laws of the reducing theory (statistical mechanics). For example, while thermodynamics talks of temperature and entropy, statistical mechanics does not. Some kind of connection between the predicates appearing in the reduced theory and those in the reducing theory (provided by so-called "bridge laws") is required so that a derivation of the laws of the one from the laws of the other will be possible. These bridge principles are essential, because explanation and reduction are conceived to be arguments in a formal language. They are linguistic relations that obtain between the theories understood as sets of sentences in that formal language.

As I mentioned, it is commonplace to read that bridge laws or connections can be found in the thermodynamics/statistical mechanics case. For instance, one often sees that "temperature" in thermodynamics is to be identified with "mean molecular kinetic energy." This is taken to be a paradigm example of the sort of bridge law Nagel demands for reduction.

There are many reasons to be sceptical that a Nagelian or neoNagelian reduction of thermodynamics to statistical mechanics is possible. One worry concerns the status of the bridge laws. In one sense they seem to be statements of definition. But surely they cannot be logical connections - statements true solely in virtue of the meanings of the terms and, perhaps, knowable merely by reflection upon those meanings. Another possibility is that the bridge principles are stipulated conventions or coordinative definitions. A third possibility is that they express factual claims that essentially have the 
status of physical hypotheses. [10, p. 354]

In the context of the reduction of thermodynamics these worries become acute. The "temperature equals mean molecular kinetic energy" bridge law identifies a fundamentally nonstatistical quantity with a fundamentally statistical quantity. How is this supposed to work? Of course, this problem will arise as well for other strictly thermodynamic predicates such as entropy.

Actually, the situation is even more dire. Even terms such as "pressure" require appropriate bridge laws. Surely it is correct to associate in some way the thermodynamic pressure with some function of the number of collisions per unit area upon the walls of the container. But which function? As Sklar points out,

[t]here is, for a particular sample of gas at equilibrium, the actual momentum transferred by the molecules ..., and there is its average value per unit area per unit ...time. On the other hand, there is the quantity calculated for an ensemble of similarly constituted systems ..., or, alternatively by looking for the most probable value of the relevant quantity in the ensemble. Whereas the former sort of pressure, the feature of the individual system, will be expected to fluctuate, the latter kinds of ensemble quantities ..., will, of course, not. Here fluctuations will show up as assimilated into the ensemble description by the calculation of averages or most probable values for quantities, but the averages themselves are not the sort of things to fluctuate. [14, pp. 349-350]

From the point of view of these concerns about the philosophical understanding of theory reduction, it is natural to interpret Gibbs' famous chapter XIV, "Discussion of Thermodynamic Analogies" to be an investigation into the nature and status of the bridge laws required for the reduction of thermodynamics to statistical mechanics. Sklar, following the above discussion of the difficulties encountered in trying to identify thermodynamic concepts with statistical mechanical concepts, says the following:

It should not surprise us that Gibbs, when he came to associate ensemble quantities with thermodynamic quantities in Chapter XIV of his book, spoke of the "thermodynamic analogies" when he outlined how thermodynamic functional interrelations among 
quantities were reflected in structurally similar functional relations among ensemble quantities. He carefully avoided making any direct claim to have found what the thermodynamic quantities "were" at the molecular dynamical level. [14, p. 350]

Gibbs was surely cautious and he did care about the connections between thermodynamics and statistical mechanics. But I think that this may not be the most fruitful way to think about his caution or his approach to intertheoretic relations.

\section{Gibbs' Caution}

In the Preface to Elementary Principles in Statistical Mechanics Gibbs asserts that the laws of statistical mechanics for conservative systems of finite degrees of freedom are exact. [6, pp. vi-vii] Further,

[t]his does not make them more difficult to establish than the approximate laws for systems of a great many degrees of freedom, or for limited classes of such systems. The reverse is rather the case, for our attention is not diverted from what is essential by the peculiarities of the system considered, and we are not obliged to satisfy ourselves that the effect of the quantities and circumstances neglected will be negligible in the result. [6, p. vii]

Forty odd years later, Khinchin, in the context of discussing Gibbs' priority in the "systematic exposition of the foundations of statistical mechanics," notes that

[I]t was precisely the necessity of a statistical foundation for the general laws of thermodynamics that produced trends which found their expression in the construction of statistical mechanics. To avoid making any special hypotheses about the nature of the particles it became necessary in establishing a statistical foundation to develop laws which had to be valid no matter what was the nature of those particles (within quite wide limitations). $[9$, p. 3]

I think that Gibbs was the one of the first to appreciate the idea that sometimes, in the investigation and understanding of general principles or 
laws, too much attention to the details can get in the way. ${ }^{1}$ Comparatively speaking, we are able to easily formulate the laws of statistical mechanics because our "attention is not diverted from what is essential."

Gibbs discusses a number of reasons why it is important to avoid making assumptions or hypotheses about the nature of the particles or systems under investigation. One of these reasons might be considered epistemic in nature, another might be taken to be "pragmatic," and a third is of fundamental theoretical significance. Let me discuss these in turn.

In the preface to Elementary Principles Gibbs discusses the relationships between empirical thermodynamics, rational thermodynamics and statistical mechanics. He says

[t]he laws of thermodynamics, as empirically determined, express the approximate and probable behavior of systems of a great number of particles, or, more precisely, they express the laws of mechanics for such systems as they appear to beings who have not the fineness of perception to enable them to appreciate quantities of the order of magnitude of those which relate to single particles $\ldots[6$, p. vi $]$

Later on in the chapter on thermodynamic analogies he asserts that "all that is really necessary to establish the science of thermodynamics on an a priori basis" is to show by "a priori reasoning that for such systems as the material bodies which nature presents to us, [the mechanical definitions of temperature and entropy] hold with such approximation that they are sensibly true for human faculties of observation." [6, p. 166]

The point here is that it is possible to establish a connection between thermodynamic principles and mechanical principles that is sufficient to satisfy us, given our limited observational capabilities, without having to make detailed hypotheses about the nature of the system being investigated. Unfortunately, as soon as we realize that all we are establishing is the connection relative to some degree of approximation, we will feel unsatisfied:

Yet we will naturally desire to find the exact expression of those principles of which the laws of thermodynamics are the approximate expressions. A very little study of the statistical properties of conservative systems of a finite number of degrees of freedom

\footnotetext{
${ }^{1}$ This is, in fact, one of the points Khinchin is making.
} 
is sufficient to make it appear, more or less distinctly, that the general laws of thermodynamics are the limit toward which the exact laws of such systems approximate, when their number of degrees of freedom is indefinitely increased. [6, p. 166]

Gibbs' recognition of this limiting behavior represents the third, theoretically fundamental, reason for eschewing detailed hypothesizing about the nature of the systems under investigation. Before turning to a discussion of this, let me briefly describe what I have called the pragmatic reasons.

Gibbs was working at a time in which physics was beginning a period of what Kuhn called "extraordinary" science. The classical paradigm was beginning to succumb to a number of anomalies whose ultimate resolution required the quantum theory. Gibbs was well aware of these anomalies and they provided pragmatic reasons for him to be sceptical of detailed hypotheses about the nature of the systems. For example, Gibbs refers to the problem of a gas composed of diatomic molecules for which according to the equipartition theorem there should be six degrees of freedom sharing the energy, whereas experiments on the specific heat of such a gas indicate that only five degrees of freedom apparently share in the energy. The other example he mentions in several places concerns the phenomenon of radiant heat. Here the problem is that the assumption that we are dealing with system of finite degrees of freedom, does not appear to be adequate for the explanation "of the properties of bodies." [6, p. 167]

These theoretical and experimental anomalies clearly contributed to Gibbs' inclination not to formulate specific hypotheses about the nature of various systems. Surely, they were significant factors relating to his caution toward providing "true" as opposed to merely analogical connections between thermodynamic concepts and statistical mechanical concepts.

Nevertheless, as I noted, there is a more fundamental reason to eschew the making of specific hypotheses. Paradoxically, it is also a reason, at least in retrospect, for thinking that his caution and repeated disclaimers ultimately were not completely warranted.

As I noted, Gibbs was aware that the approximate a priori justification of the thermodynamic laws - the one that depends upon our inability to appreciate quantities on the order of magnitude of single particles - is not entirely satisfactory. One quickly and naturally wants more. We desire to find the "exact expression of those principles of which the laws of thermodynamics are the approximate expression." [6, p. 166] To some extent one can find such 
exact expressions by studying statistical mechanical principles in the limit of increasing numbers of degrees of freedom. This limit is now often referred to as the "thermodynamic limit."

Gibbs' take on the situation is nicely summarized by the following passage:

But although these difficulties [the anomalies just discussed] seem to prevent, in the present state of science, any satisfactory explanation of the phenomena of thermodynamics as presented to us in nature, the ideal case of system of a finite number of degrees of freedom remains as a subject which is certainly not devoid of a theoretical interest, and which may serve to point the way to the solution of the far more difficult problems presented to us by nature. And if the study of the statistical properties of such systems give us an exact expression of laws which in the limiting case take the form of the received laws of thermodynamics, its interest is so much the greater. [6, p. 167 $]^{2}$

\section{Limiting Reductions/Limiting Relations Be- tween Theories}

So Gibbs realized that the thermodynamic limit will play a crucial role in establishing a connection between thermodynamics and statistical mechanics. The idea here is that the limit of statistical mechanics, as the number of degrees of freedom goes to infinity, should yield the continuum thermodynamic theory. Physicists often express this connection as an instance of reduction, though not in the same way as Nagel and his followers. On the physicists' view statistical mechanics reduces to thermodynamics (in the appropriate limit). Schematically we can represent this conception of reduction as follows:

$$
\lim _{\epsilon \rightarrow 0} T_{f}=T_{c} .
$$

\footnotetext{
${ }^{2}$ From Gibbs' point of view exact expressions are those statistical mechanical expressions involving the actual finite sums corresponding to the actual finite number of particles in the system under investigation. From a more contemporary point of view, the notion of an "exact" expression has a different meaning: In that context one obtains exact or precise results only by taking the thermodynamic limit where our inability to discern the precise number of particles becomes irrelevant, and where sharp, as opposed to fluctuating, values emerge.
} 
In the present case we can (somewhat sloppily) let $\epsilon=1 / N$ where $N$ is the number of particles, and take $T_{f}$ to be statistical mechanics and $T_{c}$ to be thermodynamics.

Philosophers, as we have seen, typically talk of reduction going the other way - that is, thermodynamics reduces to statistical mechanics via deductive derivation of the laws of the former from those of the latter. On my view, these two conceptions of reduction are related to one another. In fact, I believe that those cases where a physicists' limiting reduction holds - that is, where equality obtains in schema (1) - are cases for which the philosophers' conception of derivational reduction will likely hold as well. This is because we can take the limiting relations as providing us with something like the bridge laws appropriate for Nagel-like reduction. ${ }^{3}$

But limiting reductions in which the equality of schema (1) hold are, in fact, rare. Schema (1) will fail when the limiting relation is singular. That is to say, that the limiting behavior as $\epsilon \rightarrow 0$ is qualitatively different than the behavior at the limit when $\epsilon=0$. Singular limits do not mean that there are no interesting connections between the theories. Usually, just the opposite. But in such cases I think it is best to give up talk of "reduction" altogether and to speak instead of "intertheoretic relations."

Now I do not believe that the historical evidence is sufficient to enable us to decide whether Gibbs held a philosophical conception of reduction as deductive derivation or whether he opted for the physicists' limiting sense of reduction. I doubt that he conceptualized the issues in this way, and there are places where, with hindsight, he seems to be talking of each. Nevertheless, I would like to investigate the fruitfulness of the approach to intertheoretic relations that focuses on the importance of limits and asymptotic connections between theories.

I will consider two problem or issues, one of which was explicitly mentioned by Gibbs and is now known as the problem of the equivalence of ensembles. The second, while nowhere mentioned in Elementary Principles, is important and Gibbs was surely aware of it. This is the issue of the existence and explanation of critical phenomena and phase transitions. In fact, I want to argue that the two problems are intimately related to one another. They can both be treated using a similar strategy based upon some deep limit theorems from probability theory. Furthermore, I hope to show that

\footnotetext{
${ }^{3}$ However, these will typically be mathematical relations and not universal biconditionals of the sort Nagel and others typically talk about.
} 
the fact that these two problems are related is relevant to the possibility of realizing some kind of reductive relation between thermodynamics and statistical mechanics after all. More cautiously, perhaps, I intend to show that it is possible to assert something stronger than a mere Gibbsian analogy between certain concepts in the two theories.

\section{The Equivalence of Ensembles}

As is well known, Gibbs' canonical ensemble is appropriate for representing equilibrium systems that can be considered to be in thermal contact with a large heat bath at a fixed temperature. Here the idea is that the each member of this ensemble remains at constant energy but that the members take on all possible values for energy. The demand that this ensemble represents statistical equilibrium is the demand that the probability density $\rho(q, p)$ be invariant over a region in phase space under its dynamical evolution. Any function of phase that is constant along a phase space trajectory (hence any function of the energy) satisfies this demand, but Gibbs argues that one such function, in particular, has special features worthy of representing thermodynamic equilibrium:

$$
\rho=e^{\frac{\psi-\epsilon}{\Theta}} .
$$

Here $\epsilon$ is the energy of a system and $\psi$ and $\Theta$ are constants. Gibbs argues that one should take the negative of the "index of probability":

$$
-\log \rho=-\frac{\psi-\epsilon}{\Theta}
$$

to be analogous to the thermodynamic entropy and the modulus $\Theta$ to be analogous to the temperature. Furthermore, Gibbs argued that the deviations in energy from the mean value for energy in the ensemble should be small. In fact, he claims that those deviations should be "of the same order of magnitude as the reciprocal of the number of degrees of freedom, and therefore to human observation the individual values are indistinguishable from the average values when the number of degrees of freedom is very great." [6, p. 168] In such a situation the deviations in the index of probability from its mean will also be negligible in this operational sense.

Gibbs also discusses the microcanonical ensemble ${ }^{4}$ which is appropriate for representing equilibrium systems that can be considered to be thermally

\footnotetext{
${ }^{4}$ I ignore here the third important ensemble of Gibbs: The grand canonical ensemble
} 
isolated from the rest of the world. Here we have a collection of systems that all have the same energy. In such an ensemble the quantity

$$
\log V
$$

is the analog of the thermodynamic entropy where $V$ is the "size" or "volume" (in the natural measure) of the phase space region to which the possible microstates of the systems are confined by macroscopic constraints. The analog of temperature in the microcanonical ensemble is the quantity

$$
\frac{d \epsilon}{d \log V}
$$

Gibbs says

[w]e have thus precisely defined quantities, and rigorously demonstrated propositions, which hold for any number of degrees of freedom, and which, when the number of degrees of freedom $(n)$ is enormously great, would appear to human faculties as the quantities and propositions of empirical thermodynamics. [6, p. 169]

Nevertheless, it is well known that different quantities may approach the same form in some appropriate limit, say, $n \rightarrow \infty$.

There may be therefore, and there are, other quantities [other than the modulus, $\Theta$, and the index of probability, $\frac{\psi-\epsilon}{\Theta}$,] which may be thought to have some claim to be regarded as temperature and entropy with respect to systems of a finite number of degrees of freedom. [6, p. 169]

Of course, one such set of quantities are those derived from the microcanonical distribution: Equations (5) and (4), respectively.

Gibbs takes the fact that different statistical physical quantities apparently have equal claim to be the analogs of thermodynamic temperature and entropy to be problematic. It is yet another reason to resist claims to have found the (statistical) mechanical definition of those quantities. From a more modern perspective, however - one which recognizes explicitly the essential role played by the thermodynamic limit - this can very well be considered

which is appropriate for the description of systems that can exchange particles with the environment. 
a virtue. The idea here is that the equivalence of ensembles (to the extent that it can actually be demonstrated) is evidence of a kind of universality. The thermodynamic phenomenology obtains regardless of the exact statistical mechanical details.

One can see that this is to be expected by considering briefly Khinchin's program for statistical mechanics where the central limit theorem of probability plays a crucial role. Khinchin focuses on specific kinds of functions defined on phase space - so-called "sum functions" - having the form

$$
S(n)=\sum_{i=1}^{n} S_{i} .
$$

He employs the Central Limit theorem to show that the dispersions of the suitably normalized sum functions will, in the thermodynamic limit $(n \rightarrow \infty)$, be distributed according to the normal or Gaussian distribution. Thus we should expect that phase functions of the right form - those that presumably represent thermodynamic quantities - will be peaked around their mean values with root mean square deviations proportional to $n^{1 / 2}$.

But the real question is why the Gaussian distribution should play such a fundamental role. Why should it emerge as the limiting distribution for statistical systems in equilibrium virtually regardless of the nature of the systems' molecular details and regardless of whether it is best represented using the canonical or the microcanonical distributions of Gibbs? To answer this, I will sketch an argument from probability theory that employs the renormalization scheme developed by Kadanoff, Fisher, and Wilson. The connections between renormalization in statistical mechanics and probability theory have been stressed in a series of articles in the 1970's by Sinai, Cassandro, and, most forcefully, by Jona-Lasinio $[2,13,11,3,8]$. My presentation here follows Sinai's argument in [12, Lecture 15].

Suppose we have a sequence of random variables $S_{i}$ such as spins on a one dimensional lattice. Suppose further that the $S_{i}$ 's have finite second moments and are independent and identically distributed with the mean values, $E\left(S_{i}\right)=0 .{ }^{5}$ Consider a subsequence of the integers, $n_{p}=2^{p}$, and define the random variable, $S_{p}$ - a sum function of the spins - as follows:

$$
S_{p}=\frac{1}{2^{p / 2}} \sum_{i=1}^{2^{p}} S_{i} .
$$

\footnotetext{
${ }^{5} E(\cdot)$ represents the expectation. The assumption of independence will be relaxed later.
} 
It follows that

$$
S_{p+1}=1 / \sqrt{2}\left(S_{p}^{\prime}+S_{p}^{\prime \prime}\right)
$$

where

$$
S_{p}^{\prime}=\frac{1}{2^{p / 2}} \sum_{i=1}^{2^{p}} S_{i} \quad \text { and } \quad S_{p}^{\prime \prime}=\frac{1}{2^{p / 2}} \sum_{i=2^{p}+1}^{2^{p+1}} S_{i} .
$$

For example, let $p=2$ then

$$
\begin{aligned}
& S_{2}^{\prime}=\frac{1}{2} \sum_{i=1}^{4} S_{i}=\frac{1}{2}\left(S_{1}+S_{2}+S_{3}+S_{4}\right) \\
& S_{2}^{\prime \prime}=\frac{1}{2} \sum_{i=5}^{8} S_{i}=\frac{1}{2}\left(S_{5}+S_{6}+S_{7}+S_{8}\right) .
\end{aligned}
$$

From (6) we have

$$
S_{3}=\frac{1}{2^{3 / 2}}\left(S_{1}+\cdots+S_{8}\right)
$$

But we see from (8) that $\left(S_{1}+S_{2}+S_{3}+S_{4}\right)=2 S_{2}^{\prime}$ and from (9) that $\left(S_{5}+S_{6}+S_{7}+S_{8}\right)=2 S_{2}^{\prime \prime}$. Hence,

$$
S_{p+1}=S_{3}=\frac{1}{\sqrt{2}}\left(S_{2}^{\prime}+S_{2}^{\prime \prime}\right) .
$$

So, $S_{p}^{\prime}$ and $S_{p}^{\prime \prime}$ are independent and identically distributed random variables. The probability distribution for the sum of two independent random variables $\xi_{1}, \xi_{2}$, (that is, for the random variable $\xi=\xi_{1}+\xi_{2}$ ) is given by the convolution of their individual distribution functions. Thus if $F_{1}$ is the distribution function for $\xi_{1}$ and $F_{2}$ is the distribution function for $\xi_{2}$, then

$$
F_{1,2}(x)=\int_{-\infty}^{\infty} F_{1}(x-u) d F_{2}(u)
$$

is the distribution function for the random variable $\xi$. Now, the random variables $S_{p}^{\prime}$ and $S_{p}^{\prime \prime}$ are not only independent but they are also identically distributed. Let $F_{p}$ be the distribution function for $S_{p}$-equation (6) which is this common distribution function. Then from (10) we have

$$
F_{p+1}=\int_{-\infty}^{\infty} F_{p}(\sqrt{2} x-u) d F_{p}(u),
$$


which is the distribution function for the random variable $S_{p+1}$ (recall (7)). It follows that for any $p$, the distribution function $F_{p}$ for the variable $S_{p}$ can be obtained by iterating this nonlinear convolution operation starting from the distribution function $F_{0}$ for $S_{0}$ - the random variable for an individual spin. Thus, let

$$
T F=\int_{-\infty}^{\infty} F(\sqrt{2} x-u) d F(u)
$$

for any distribution function $F$ then $F_{p+1}=T F_{p}$ and $F_{p}=T^{p} F_{0}$. The operator $T$ is the analog, for the current case, of the renormalization group operator.

It can be shown that the Gaussian distribution,

$$
G(x)=\frac{1}{\sqrt{2 \pi}} \int_{-\infty}^{x} e^{-\frac{u^{2}}{2}} d u
$$

is a fixed point of the transformation $T$. In other words, $T G=G$. The emergence and the ubiquity of the Gaussian distribution is related to the stability of this fixed point in the space of distributions and that stability can be investigated by examining the stability of the linearization, $L$, of the nonlinear operator $T$ in the neighborhood of the fixed point $G$.

Sinai shows that the linear operator $L$ at the point $G$ is given by

$$
L h=\frac{\sqrt{2}}{\sqrt{\pi}} \int_{-\infty}^{\infty} h\left(u+\frac{x}{\sqrt{2}}\right) e^{-\frac{u^{2}}{2}} d u .
$$

One then determines the spectrum and eigenfunctions of $L$. It turns out that the eigenfunctions are Hermite polynomials $h=P_{k}(x)$ with eigenvalues

$$
u_{k}=2^{1-\frac{k}{2}}
$$

for $k=0,1,2, \ldots$ It follows that $u_{0}, u_{1}>1, u_{2}=1$, and for all $k>2, u_{k}<1$. Thus, the "directions" $P_{0}$ and $P_{1}$ are unstable, $P_{2}$ is marginal and all other directions are stable. This analysis tells us that the fixed point $G$ is stable for linear approximation with respect to perturbations that lie in the class of distribution functions $F$ with zero expectation and finite variance.[12, p. 132] And so, a large class of distribution functions will behave just like the Gaussian in the limit $n_{p} \rightarrow \infty$. This class of distributions, in the jargon of renormalization group theory, is a "universality class."

The upshot of this analysis for the problem of the equivalence of ensembles is that we must expect those finite systems distributed according to the 
microcanonical distribution, and those distributed according to the canonical distribution, to behave in the same way as the number of their components gets large. In fact, we can use the Gaussian distribution to calculate the dispersions of various quantities from their means for large finite systems of either type. A further consequence of this form of argument is that in order to solve certain problems regarding universal behavior, one need only work with the most convenient "system" in the relevant universality class. Nigel Goldenfeld calls such a system a "minimal model" and asserts that it is a model that "most economically caricatures the essential physics." [7, p. 33] In the current context, Gibbs' insistence that for most problems it is easier to calculate using the canonical distribution, is an indication that the canonical distribution may be considered to be a minimal model. ${ }^{6}$

While Gibbs took the existence of the different quantities associated with the different ensembles - each apparently having equal claim to be analogs of thermodynamic temperature and entropy - to be a problem, from the current perspective, we should take their existence to be a virtue: In the thermodynamic limit, we can demonstrate the equivalence of the ensembles, and we can show that calculations with either ensemble will yield the thermodynamic phenomenology that we are after. On this way of thinking, the question of what quantity is to be offered as the definition of thermodynamic entropy, say, is not really well-formed. In the context of intertheoretic relations, the study of the thermodynamic limit renders such a question moot.

I will say more about this shortly. But first we must address some obvious questions and objections to the argument presented above. On the one hand, Sinai's demonstration of the emergence and ubiquity of the Gaussian demonstration assumes that the random variables are independent. To what extent is this assumption legitimate for real thermodynamic systems? On the other hand, it also assumes that we are working with a particular subsequence of the integers, $n_{p}=2^{p}$. What is the role of this assumption? Finally, how does this entire argument, one which is purely mathematical relate to real systems?

Let's take up the second question first. It is absolutely crucial. The particular subsequence $n_{p}=2^{p}$ was chosen so that we would have the right normalization factor for the sum function - for the random variable $S_{p}$. One of Kadanoff's main insights was that as we sum over blocks of spins (which is, in effect, what we are doing by considering sum functions) and then try to

\footnotetext{
${ }^{6}$ See [1] for a discussion of minimal models and asymptotics.
} 
replace these sums with individual blocks-spins (averages perhaps) we need to try to keep the (Hamiltonian) structure looking the same at these different scales. Equivalently, we need to make sure that the partition function for the original lattice is similar to that for the lattice of block-spins. One of the main difficulties of employing the renormalization techniques in investigating the behavior of model systems is to determine exactly how one needs to rescale (or normalize) a collective variable like $\sum S_{i}$ so as to arrive at reasonable results. In fact, this is largely the art of the renormalization group approach. In the example above, this difficult problem is taken care of by employing that very special subsequence of the integers to form our "blocks" of spins. This allows us to write down, without any difficulty, the "renormalization group equation," (12), for our distribution functions. In effect, the use of the special subsequence of the integers gives us the proper normalization for the variables $S_{p}$ and determines the factor $\sqrt{2}$ that appears in equation (12). In physical applications, however, we do not have the luxury of beginning with a solution to how the block-spins are to be normalized.

The first problem concerns the fact that the demonstration of the universality of the Gaussian distribution relies upon the fact that the random variables considered are independently distributed. Of course, this is far from true even for the simplest ofthermodynamic systems: For example, the kinetic energies of molecules in an isolated gas are correlated with one another as a result of collisions and the fixed total energy of the gas. This poses a problem for Khinchin's program - one of which he was well-aware. He tries to deal with it by insisting that we must really think of the particles (in a real gas) as being "only approximately isolated energetically components." When being precise, however, we must allow for correlations between the components that would, strictly speaking, block the ability to use the central limit theorem. He says,

inasmuch as forces of interaction between particles manifest themselves only at very small distances, such mixed terms in the expression of energy, representing mutual potential energy of particles, will be (in the great majority of points of the phase space) negligible as compared with the kinetic energy of particles or with the potential energy of external fields. In particular, they will contribute very little in evaluating various averages. ... However, these mixed terms that are neglected, from the point of principle play a very important role, since it is precisely their presence 
that assures the possibility of an exchange of energy between the particles, on which is based the whole of statistical mechanics. [9, pp. 43-43]

This is hardly a satisfying response to the problem of interaction or correlation: "The entire success of statistical mechanics depends upon such interactions, but we need to ignore them completely because they will be small."

In fact, though, it is possible to generalize the above argument for the universality of the Gaussian distribution to sequences of dependent random variables, provided that the dependence is not too strong. This is a truly remarkable feature of the central limit theorem. One can show that limiting Gaussian behavior is to be expected even for a large class of dependent random variables. Without going into details, let me discuss the degree of robustness of such central limiting behavior and when one might actually expect it to fail. To this end, consider once again a sequence of independent and identically distributed random variables $S_{i}(i=1,2, \ldots){ }^{7}$ Suppose the $S_{i}$ 's are centered at the origin and have finite variance. This means, respectively, that

$$
\begin{aligned}
E\left(S_{i}\right) & =0 \\
E\left(S_{i}^{2}\right) & =\sigma^{2} .
\end{aligned}
$$

Consider the sum function $S_{N}=\sum_{i=1}^{N} S_{i}$. The mean square deviation of $S_{N}$ is given by

$$
E\left(S_{N}^{2}\right)=\sum_{i=1}^{N} E\left(S_{i}^{2}\right)=N \sigma^{2} .
$$

If we now suppose that the variables $S_{i}$ are not independent (13) needs to be modified to reflect the contribution of correlations to this average. So write

$$
E\left(S_{N}^{2}\right)=\sum_{i, j=1}^{N} E\left(S_{i} S_{j}\right)=\sum_{i, j=1}^{N} R(i, j)
$$

where $R(i, j)$ is the correlation function of the sequence. In physical applications we typically consider only stationary sequences which means that there

\footnotetext{
${ }^{7}$ Here I follow the discussion in [3, pp. 914-916].
} 
is kind of translation invariance - no privileged $i$ in the sequence. Assuming stationarity we get

$$
E\left(S_{N}^{2}\right)=\sum_{i, j=1}^{N} R(i, j)=N R(0)+2 \sum_{l=1}^{N}(N-l) R(l) .^{8}
$$

The first term reduces to $N \sigma^{2}$ when the variables are independent and so represents the contributions to the fluctuations of the variable $S_{N}$ if the sequence had been independent with variance $R(0)$. The second term is nonzero when correlations between the $S_{i}$ exist. Cassandro and Jona-Lasinio consider several possibilities for the behavior of $R(l)$. [3, p. 915] The two most relevant to our discussion are the following:

$$
\sum_{l=1}^{N} R(l) \stackrel{N \rightarrow \infty}{\longrightarrow} \bar{R}<\infty
$$

and

$$
\sum_{l=1}^{N} R(l) \stackrel{N \rightarrow \infty}{\longrightarrow} \infty
$$

When (16) holds it is clear that for large $N$, the mean square deviation of $S_{N}, E\left(S_{N}^{2}\right)$, will still be proportional to $N$ for large $N$ and the $\sqrt{N}$ fluctuation law will still hold. Cassandro and Jona-Lasinio say that in this situation the variables $S_{i}$ are "weakly dependent." Weakly dependent systems of variables satisfy the condition of "strong mixing" that, physically, is related to an exponential decay of correlations with distance. In such cases it is possible to show that the distribution functions of these weakly dependent random variables will flow, under the transformation $T$ to the Gaussian fixed point. Thus, this answers the worry about using the Central Limit theorem in situations where independence fails. That is to say, central limiting behavior is quite robust and the independence condition in the theorem as it is usually formulated can be considerably weakened. There is, therefore, no need to accept Khinchin's somewhat handwaving and inconsistent solution to the problem of interacting components.

When (17) holds

$$
\frac{E\left(S_{N}^{2}\right)}{N} \rightarrow \infty
$$

\footnotetext{
${ }^{8}$ Stationarity entails that $l$ will be the same for any pairs $(i, j)$ equal separation $(|i-j|)$.
} 
for large $N$ and the fluctuations will be greater than $\sqrt{N}$. This is the situation of "strongly dependent" variables. Now if we think of the variables $S_{i}$ as spins on a $d$-dimensional lattice $\mathbb{Z}^{d}$, we expect stationarity to hold which is to say that $R(\mathbf{i}, \mathbf{j})=\mathbf{R}(\mathbf{i}-\mathbf{j})$ where $\mathbf{i}$ and $\mathbf{j}$ are $d$-dimensional lattice vectors. In such a case, (17) is expressed as follows:

$$
\sum_{l \in \mathbb{Z}^{d}} R(l)=\infty
$$

This says that the correlation length between spins on the lattice is infinite. In statistical mechanics this means that the system is at a point of second order phase transition - a critical point. This takes us directly to what very well may be another fundamental reason for Gibbs' caution in identifying thermodynamic quantities with (statistical) mechanical features of systems - the existence of critical phenomena.

Before turning to this let me briefly take stock of the status of reductive relations between thermodynamics and statistical mechanics up to this point. The argument of this section is designed to show that, pace Gibbs, we should not really worry about the fact that different "precisely defined quantities" have some kind of equal claim to be "regarded as as temperature and entropy with respect to systems of a finite number of degrees of freedom." $[6$, p.169] In fact, the canonical and microcanonical distributions will yield the same results in the thermodynamic limit. They both lie within the basin of attraction of the Gaussian distribution - in the Gaussian universality classunder the probabilistic version of the renormalization group transformation sketched above. So Gibbs is being overly cautious from the point of view being advocated here.

By considering the thermodynamic limit in the context of intertheoretic relations, we do in effect demonstrate the existence of a limiting connection between statistical mechanics and thermodynamics akin to the physicists' schema (1) for reduction. That is to say that schema (1) holds where $T_{f}$ is statistical mechanics and $T_{c}$ is thermodynamics and $\epsilon=1 / N .^{9}$ The proof of the equivalence of the microcanonical and the canonical ensembles in the thermodynamic limit, together with the probabilistic renormalization group argument given above, provides the required limiting connections between the two theories. Given this, we should expect something like a philosopher's

\footnotetext{
${ }^{9}$ More precisely, the thermodynamic limit is the limit in which $N \rightarrow \infty$, the volume $V \rightarrow \infty$ with the constraint that the density $N / V \rightarrow$ constant.
} 
derivational reduction to obtain. However, contrary to the Nagelian point of view which requires bridge laws identifying a thermodynamic quantity with a unique statistical mechanical quantity, we instead are able only to provide an association between the thermodynamical properties, such as temperature and entropy, and a universality class of statistical mechanical structures.

\section{Critical Phenomena}

While we seem to have been successful in finding a limiting connection between statistical mechanics and thermodynamics, there are prima facie reasons to believe that such a reduction cannot hold in general. This is because, the limiting schema (1) fails to hold for every thermodynamic phase. The existence of phase transitions and critical points guarantee that a smooth limiting relationship between the theories cannot hold everywhere.

Physically this failure of smooth limiting behavior is related to the divergence of various quantities at the critical points. For instance, at a critical point $\left(P_{c}, V_{c}, T_{c}\right)$ the compressibility

$$
\kappa \equiv\left[-V\left(\frac{\partial P}{\partial V}\right)_{T}\right]^{-1}
$$

becomes infinite.

From a modern perspective this failure can be related to the inequivalence of the microcanonical and canonical ensembles at certain phases. Giovanni Gallavotti takes this to be a virtue of statistical mechanics rather than a defect.

... $[R]$ ather than being an obstacle to the microscopic formulation of thermodynamics, [this inequivalence] shows the possibility that statistical mechanics can be a natural frame in which to study the phase transition phenomenon. ...

We can therefore conclude, in the case ... of the canonical and microcanonical ensembles, that they provide equivalent descriptions of the system thermodynamics in the correspondence of the parameter values to which no phase transition is associated. In the other cases, the possible nonequivalence cannot be considered a defect of the theory, but it can be ascribed to the fact that, 
when equivalence fails, the elements of the two statistical ensembles that should be equivalent are not because they describe two different phases that may coexist (or different mixtures of coexisting phases). [4, p. 74-75]

Now Gibbs' work on thermodynamics prior to writing Elementary Principles in Statistical Mechanics, focused largely on representing (geometrically and analytically) different phases of thermodynamic systems including places of phase transitions and critical points. ${ }^{10}$ It is inconceivable to me that he was not aware of the problem that the existence of critical states raises for identifying precisely defined (statistical) mechanical properties with thermodynamic properties. That is, I think one major factor behind Gibbs' caution - a major reason he allowed himself to speak only of thermodynamic analogies and not of identities - was the recognition that such identities will clearly fail at critical phases.

It is, therefore, extremely odd that Gibbs makes no mention of phase transitions and critical phenomena in Elementary Principles in Statistical Mechanics. This omission has been noted elsewhere by A. S. Wightman. Wightman states that

[t]here is one aspect of the thermodynamic limit that Gibbs does not emphasize. That is the appearance of phase transitions between distinct thermodynamic phases. Such sharp phase transitions do not occur in finite systems .... It is only in the thermodynamic limit that sharp phase transitions appear. A little more pedagogical zeal by Gibbs on this point would have saved some of the generations that followed considerable time. [15, p. 34]

It is certainly true that one can only find sharp (nonanalytic) phase transitions in the thermodynamic limit. It is also true that that limit is singular on lines of phase transitions and at critical points.

As Gallavotti has noted, the equivalence of the ensembles in the thermodynamic limit can fail, and when it does, it means that the system is at criticality. We can no longer expect Gaussian behavior to emerge as a limiting distribution in the iterative renormalization group equation (12). Some have argued that this situation is an indication that we cannot expect general

\footnotetext{
${ }^{10}$ See, for example [5] and other papers in Volume One of his Collected Works.
} 
reductive limiting relations between thermodynamics and statistical mechanics to obtain. ${ }^{11}$ However, if we take the inequivalence of the ensembles, and the existence of critical phenomena, to be two sides of the same coin, as Gallavotti suggests, then perhaps this pessimistic assessment is too quick. In this section, I would like to suggest that the probabilistic interpretation of the renormalization group provides a mathematical framework with which to investigate both sides of this coin using the same methodology. We shall see that the divergences and singularities at critical phases are not genuine obstacles to some kind general limiting (reductive?) relation between the theories after all. Rather than give up on intertheoretic reduction in this case, we just need to recognize how involved it really is, and how different the reductive relation really is from the traditional philosophical conception.

We have seen that Gaussian limiting behavior fails when the random variables $S_{i}$ are strongly dependent. This is the situation expressed by equation (17), generally, and by equation (18) for the case of spins on a $d$-dimensional lattice. Equivalently, given equation (14), this means that the variance of the distribution functions for the sum functions or block-spins is infinite. Consider a generalization (one that takes correlations into consideration) of the iterative scheme (11): ${ }^{12}$

$$
F_{p+1}=g_{N}(x, a) \int_{-\infty}^{\infty} F_{p}\left(a^{-1} x-u\right) d F_{p}(u) .
$$

When the variables are strongly dependent $g_{N}(x, a) \rightarrow g_{\infty}(x, a)$ as $N \rightarrow \infty$ and this equation will, in that limit, have non Gaussian solutions. That is, there will be limiting distributions $F^{*}$ satisfying the the fixed point equation

$$
T F^{*}=F^{*}
$$

for the relevant renormalization group transformation analogous to (12):

$$
T F=g_{\infty}(x, a) \int_{-\infty}^{\infty} F_{\infty}\left(a^{-1} x-u\right) d F_{\infty}(u)
$$

Under the assumption that a solution to this equation - a fixed point - exists, the value of the parameter $a$ will determine the critical exponent $\alpha \neq 1 / 2$ that

\footnotetext{
${ }^{11} \mathrm{I}$, for one, have been a major proponent of this point of view.

${ }^{12}$ The assumption that something like the convolution equation for distribution function of a sum of two random variables holds, is the essence of the renormalization group approach.
} 
characterizes the nature of this nonGaussian limiting distribution as well as the form of the required normalization. If we assume that the correlation $R(l)$ exhibits power law behavior $R\left(l_{\alpha}\right) \sim l^{-\alpha}$ and that $\sum_{l=1}^{N} R(l) \sim \sum_{l=1}^{N} l^{-\alpha} \rightarrow$ $\infty$ as $N \rightarrow \infty$, then from equation (15) we have

$$
E\left(S_{N}^{2}\right) \rightarrow N^{2-\alpha}
$$

as $N \rightarrow \infty$.

The point of all of this is the following: There do exist nonGaussian probability distributions that are stable limit distributions under transformations of the form (21). ${ }^{13}$ These distributions represent fixed points of the probabilistic renormalization group transformation for strongly dependent random variables. Strong dependence is exactly what we expect in real systems at criticality: It is reflected in the divergence of the correlation length. By generalizing the argument of the last section, it is possible to show that these fixed points have large basins of attraction reflecting the fact that at criticality a wide variety of distributions will all exhibit the same, nonGaussian, behavior in the limit $N \rightarrow \infty$.

In effect, this solves the critical phenomenon problem. We can explain the universality (the virtual independence of behavior from microscopic detail) of systems at criticality. The very same strategy that can be used to show that the microcanonical and canonical distributions behave like Gaussians in the thermodynamic limit is used to explain the universal nonGaussian behavior of systems at criticality.

\section{Conclusion}

The best understanding of intertheoretic relations between statistical mechanics and thermodynamics is to be had by investigating the limiting relations, both regular and singular that can be expressed in terms of schema (1). We can say (with various qualifications ${ }^{14}$ ) that away from critical points and

\footnotetext{
${ }^{13}$ Stability here means that a block random variable (a block-spin or sum function) with distribution function $F$ can be split into the sum of two block random variables of arbitrary relative size having a probability distribution of the same type; namely, $F$. Recall equation (7).

${ }^{14}$ These are, in fact, serious qualifications that may lead us ultimately to deny anything like a Nagelian reduction of the full theory of thermodynamics to statistical mechanics. See [14, Chapter Nine].
} 
phase transitions, statistical mechanics does reduce to thermodynamics in the thermodynamic limit. And, we can say that there does exist a kind of identification of certain thermodynamic quantities such as temperature and entropy with universality classes of statistical mechanical quantities. In this context - that is, from the modern perspective of the success of renormalization group arguments - we see that Gibbs need not have been so cautious regarding the status of the connections between mechanical quantities and thermodynamic quantities. The existence of the thermodynamic limit, and the demonstration of the equivalence of ensembles, provides evidence that the question of which ensemble quantity is really to be identified with thermodynamic entropy, say, may not even be an important question to ask.

On the other hand, Gibbs was an expert on phase transitions and critical phenomena. I think that he must have understood that such phenomena present a problem for connecting the two theories. In his interest to avoid saying anything false, he did not raise the issue in his book. From the point of view I have been advocating, his understanding this problem is tantamount to recognizing that the limiting relationship between statistical mechanics and thermodynamics as $1 / N \rightarrow 0$ is not everywhere regular. Despite this, the framework provided by the probabilistic interpretation of renormalization group argument indicates that it is possible to employ these techniques to connect both noncritical and critical thermodynamics to an underlying statistical mechanics. Both problems - the equivalence of ensembles and the inequivalence of ensembles - receive a unified, coherent treatment.

\section{References}

[1] R. W. Batterman. Asymptotics and the role of minimal models. The British Journal for the Philosophy of Science, 53:21-38, 2002.

[2] P. M. Bleher and Ya. G. Sinai. Investigation of the critical point in models of the type of dyson's hierarchical models. Communications in Mathematical Physics, 33:23-42, 1973.

[3] M. Cassandro and G. Jona-Lasinio. Critical point behaviour and probability theory. Advances in Physics, 27:913-941, 1978.

[4] Giovanni Gallavoti. Statistical Mechanics: A Short Treatise. Texts and Monographs in Physics. Springer-Verlag, Berlin, 1999. 
[5] J. Willard Gibbs. A method of geometrical representation of the thermodynamic properties of substances by means of surfaces. In The Collected Works of J. Willard Gibbs, chapter II, pages 33-54. Longmans, Green and Co., New York, 1928. Originally Published in Transactions of the Connecticut Academy, II pp. 382-404 Dec. 1893.

[6] J. Willard Gibbs. Elementary Principles in Statistical Mechanics: Developed with Especial Reference to The Rational Foundation of Thermodynamics. Ox Bow Press, 1981. First Published in 1902.

[7] Nigel Goldenfeld. Lectures on Phase Transitions and the Renormalization Group. Frontiers in Physics. Addison-Wesley Publishing Company, Reading, Massachusetts, 1992.

[8] G. Jona-Lasinio. The renormalization group: A probabilistic view. Il Nuovo Cimento, 26 B(1):99-119, 1975.

[9] A. I. Khinchin. Mathematical Foundations of Statistical Mechanics. Dover Publications, New York, 1949. Translation: G. Gamow.

[10] Ernest Nagel. The Structure of Science: Problems in the Logic of Scientific Explanation. Harcourt, Brace, \& World, 1961.

[11] Ya. G. Sinai. Theory of Phase Transitions: Rigorous Results. Pergamon Press, 1982.

[12] Ya. G. Sinai. Probability Theory: An Introductory Course. SpringerVerlag, Berlin, 1992. Trans.: D. Haughton.

[13] Yakov G. Sinai. Mathematical foundations of the renormalization group method in statistical physics. In G. Dell'Antonio, S. Doplicher, and G. Jona-Lasinio, editors, Mathematical Problems in Theoretical Physics, pages 303-311. Springer-Verlag, 1978.

[14] Lawrence Sklar. Physics and Chance: Philosophical Issues in the Foundations of Statstical Mechanics. Cambridge University Press, Cambridge, 1993.

[15] A. S. Wightman. On the prescience of J. Willard Gibbs. In G. D. Mostow and D. G. Caldi, editors, Proceedings of the Gibbs Symposium: Yale University May 15-17, 1989, pages 50-65. American Mathematical 
Society, American Institute of Physics, American Mathematical Society, 1989. 